\title{
Evaporation of volatile compounds in suspended-core fibers
}

\author{
J. P. Moura, ${ }^{1,2, *}$ H. Baierl, ${ }^{3,4}$ J.-L. Auguste, ${ }^{4}$ R. Jamier, ${ }^{4}$ P. Roy, ${ }^{4}$ J. L. Santos,${ }^{1,2}$ and O. Frazão ${ }^{1,2}$ \\ ${ }^{1}$ INESC TEC, Rua do Campo Alegre 687, 4169-007 Porto, Portugal \\ ${ }^{2}$ Department of Physics and Astronomy, Faculty of Sciences, University of Porto, Rua do Campo Alegre 687, 4169-007 Porto, Portugal \\ ${ }^{3}$ Optical Fiber Technology Group, Leibniz Institute of Photonic Technology, Jena 07745, Germany \\ ${ }^{4}$ Fiber Photonics Department, UMR CNRS/University of Limoges 7252, 123 Avenue Albert Thomas, 87060 Limoges Cedex, France \\ *Corresponding author: jpmoura@fc.up.pt
}

Received April 17, 2014; revised May 22, 2014; accepted May 27, 2014;

posted May 30, 2014 (Doc. ID 210317); published June 24, 2014

\begin{abstract}
A sensing configuration for fluid evaporation monitoring using a suspended-core fiber tip is proposed. Strong differences between the evaporation processes of acetone and isopropyl alcohol were observed, both in terms of the signal's intensity fluctuations and total duration. In each fluid, the main signal variations were due to changes in reflectivity inside a collapsed region of the suspended-core fiber near the spliced interface with a standard singlemode fiber. After further analysis with a wider array of substances, this configuration could, in the future, be used to detect and study the evaporation of different volatile organic compounds. (c) 2014 Optical Society of America OCIS codes: (060.2370) Fiber optics sensors; (060.4005) Microstructured fibers; (280.1545) Chemical analysis. http://dx.doi.org/10.1364/OL.39.003868
\end{abstract}

Suspended-core optical fibers (SCFs) are characterized by their nanowire-like small-diameter cores held by thin struts in the middle of large air holes [1]. By reducing the core size, an increasing fraction of the core-guided modes protrude into the air holes. Light can then strongly interact with fluids that are inserted into these holes without the characteristic frailty of thin nanowires [2]. Although hollow-core photonic bandgap fibers also have a large interaction area with the air holes, the ease of fabrication of SCFs gives them a competitive advantage when planning for industrial applications [3]

The interaction between the evanescent field and fluids inserted into microstructured fiber holes has been the object of extensive study for sensing purposes. Suspended-core fibers have been used to measure physical parameters [ $\underline{4}$ ] for Raman and fluorescence spectroscopy $[\underline{1}, \underline{4}]$ and in biological and chemical sensing. In particular, measurements of liquids have been performed either by effective refractive index changes in interferometric setups or by absorption spectrum analysis [ㄷ].

Recently, Preter et al. introduced a new technique in microstructured fibers for liquid sensing []]. Using in-line microcavities etched in single-mode fibers (SMF), the authors proposed monitoring the evaporation dynamics of volatile organic compounds as a means to identify them. They tracked the evaporation of ethanol, hexane, acetone, and a mixture of ethanol and hexane, observing clear distinctions in light loss dynamics, which effectively resulted in a volatile compound sensor.

In this work, the concepts used in microcavities are applied to suspended-core fibers in a dipping configuration. The time-dependent signal response of a suspendedcore fiber is monitored when its cavities are filled with acetone and IPA. The phenomenon was observed simultaneously with an optical microscope.

Figure 1(a) represents schematically the experimental setup. A $(0.34 \pm 0.02) \mathrm{mm}$ long suspended-core fiber segment was fusion spliced to a standard SMF. An asymmetric four-bridge silica fiber with a double-Y-shaped suspended core was used. The cross section of the micro- structured fiber can be seen in Fig. 1(b). The fiber's outer diameter is $130 \mu \mathrm{m}$ while the diameter of the air-hole cladding is $65 \mu \mathrm{m}$. The core [Fig. 1(c)] is approximately rectangular with a length of $6.5 \mu \mathrm{m}$ and a width of $1.5 \mu \mathrm{m}$.

The sensor was dipped in a volatile organic compound reservoir at room temperature, and its time-dependent evaporation dynamics were studied. To perform the interrogation, the SMF was connected to an optical circulator. Light was introduced from a $100 \mathrm{~nm}$ wide optical source centered at $1550 \mathrm{~nm}$, and the reflected signal was interrogated using an optical spectrum analyzer with a maximum resolution of $0.01 \mathrm{~nm}$. An optical microscope was used to observe the phenomenon. At rest, the sensor presents a Fabry-Perot cavity spectrum with a period of $2.7 \mathrm{~nm}$, which is in agreement with the cavity length, and an amplitude of $1.6 \mathrm{~dB}$ (Fig. 2).

Figure $\underline{3}$ shows measurements of the sensing head's reflected signal as a function of time after dipping in acetone and isopropyl alcohol (IPA). Three different measurements are displayed for each substance, which shows the reproducibility of results. Figure $\underline{4}$ displays the evaporation process as observed through the optical microscope.

In order to synchronize all measurements, the most obvious approach would be to consider the beginning of the measurement process as soon as the first signal drop is observed, i.e., right when the sensing head is dipped in the corresponding substance. However, two problems arise. First, the dipping process was performed manually. Consequently, perfect reproducibility of the time during which the sensing head is immersed in liquid could not be guaranteed, causing delay differences until the beginning of the actual evaporation process. Second, after dipping, different amounts of liquid surround the fiber tip through the formation of a droplet around the sensing head. The evaporation of this droplet can be tracked with our sensor, and it is also associated with reduced reproducibility. As such, all measurements were synchronized by setting the most abrupt signal increase as $t=0 \mathrm{~s}$, as observed in Fig. 3 . 


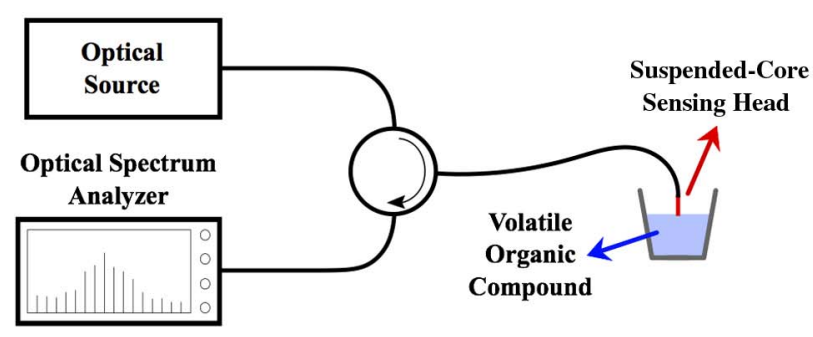

(a)

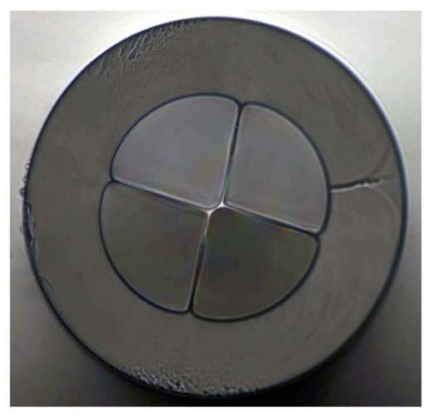

(b)

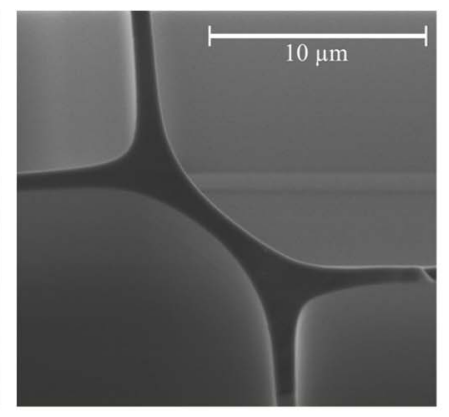

(c)

Fig. 1. (a) Diagram of the experimental setup. (b) Suspended-core fiber cross section and (c) core region.

From Fig. 3 one can observe that, although both substances shôw overall similar evaporation behavior, there exists strong differences that allow their differentiation, namely, the signals' duration and oscillations before and after $t=0 \mathrm{~s}$. The process duration is defined as the time it takes for the signal to restore its rest condition after being dipped in a volatile compound, i.e., until signal oscillations due to fluid evaporation are not detected. With this definition, acetone evaporation takes an average of $T_{\text {acet }} \approx 4.1 \mathrm{~s}$ with only minor oscillations after $t=0 \mathrm{~s}$, while IPA takes an average of $T_{\mathrm{IPA}} \approx 6.2 \mathrm{~s}$ with stronger oscillations after $t=0 \mathrm{~s}$.

One common feature in the evaporation of both substances is the existence of high- and low-signal states with strong intensity differences $\Delta$ between them. In particular, $\Delta_{\text {acet }} \approx 14 \mathrm{~dB}$ and $\Delta_{\mathrm{IPA}} \approx 15 \mathrm{~dB}$. In order to interpret the origin of these values, it is necessary to observe the structure of the fabricated sensor in closer detail. The red arrow in Fig. 4(a) indicates the presence of a defect in the SCF close to the spliced region. This corresponds to a collapse of the SCF structure due to the splicing process, which leads to the creation of connections between the fiber holes. Further evidence of this comes from observation of the evaporation process by optical microscopy through Fig. $\underline{4}$. It can be seen that, in this experimental instance, an air-acetone meniscus seems to form in one of the holes [Fig. 4(b)] and starts propagating from the outside in. As it reaches the collapsed region [Fig. $\underline{4(\mathrm{e})}$ ], the first meniscus divides into

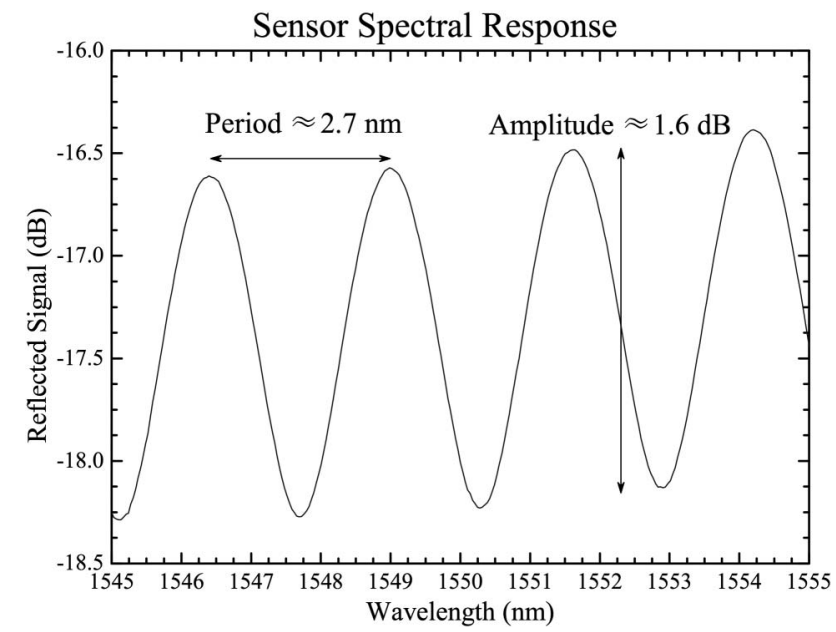

Fig. 2. Spectral response of the sensing head. several menisci in the other holes, and propagation is now from the inside out until all acetone evaporates and the sensor returns to its initial state [Fig. 4(i)].

The sensor structure is schematically represented in Fig. 5(a). Close to the spliced region a gap in the SCF core is present, which is related with the structure collapse. The indices of refraction (RI) of the SMF propagating mode $n_{1} \approx 1.45$ and of the pure silica SCF core $n_{3} \approx$ 1.44 can be considered constant throughout the sensing process. However, both the RI inside the SCF holes $n_{2}$ and outside the sensing head $n_{4}$ can take the values of $n_{\text {air }} \approx 1$ when empty or $n_{\text {acet }} \approx 1.36$ or $n_{\text {IPA }} \approx 1.38$ when filled with acetone or IPA, respectively.

With these refractive indices, three main reflections $R$ can be defined by Fresnel's equations: $R_{1}=$ $\left(\left(n_{1}-n_{2}\right) /\left(n_{1}+n_{2}\right)\right)^{2}$ on the interface between the SMF and the SCF collapsed region, $R_{2}=\left(\left(n_{2}-n_{3}\right) /\right.$ $\left.\left(n_{2}+n_{3}\right)\right)^{2}$ between the collapsed region and the SCF core, and $R_{3}=\left(\left(n_{3}-n_{4}\right) /\left(n_{3}+n_{4}\right)\right)^{2}$ between the SCF core and the external environment. An overall interpretation of the sensor's time-response can be obtained with analysis of the evolution of these reflections. The evaporation process can be divided into four different phases, which are represented by the four images in Fig. 5. The corresponding reflection values are indicated in Table $\underline{1}$ and were calculated without consideration of

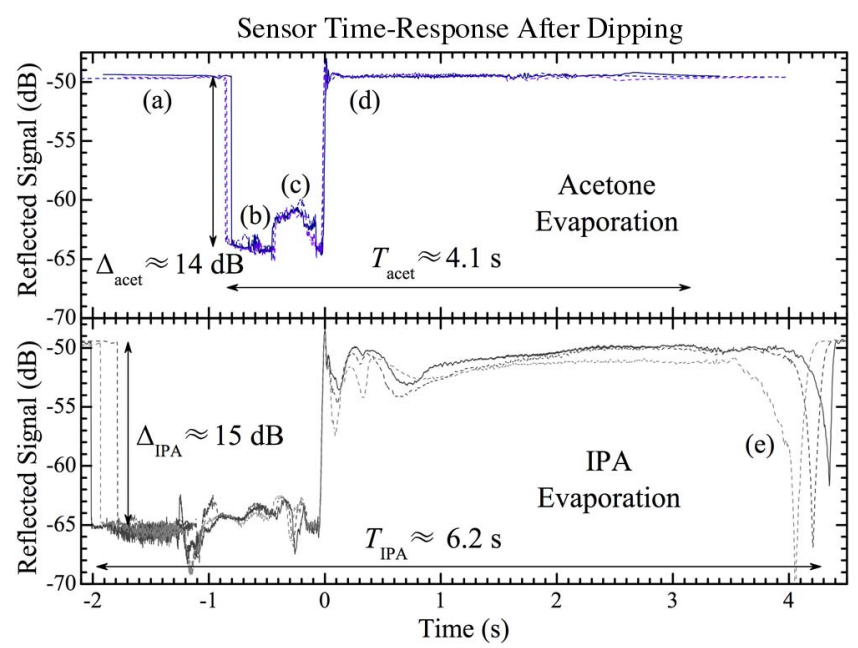

Fig. 3. Sensor's time-response after dipping in acetone and IPA. Three different measurements are shown for each substance. The letters in parenthesis indicate different evaporation phases. 


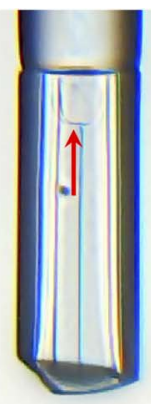

(a)

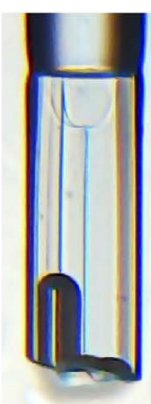

(b)

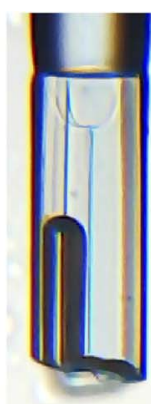

(c)

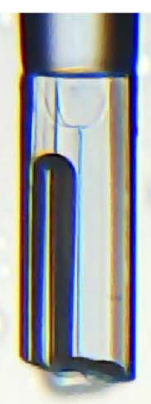

(d)

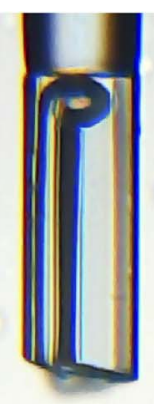

(e)

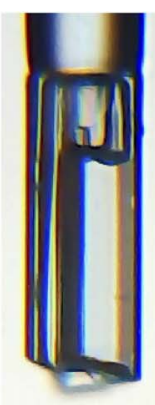

(f)

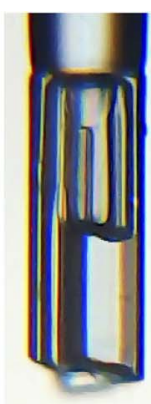

(g)

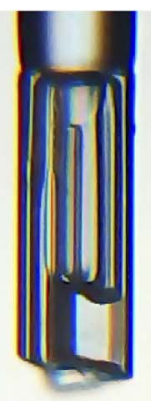

(h)

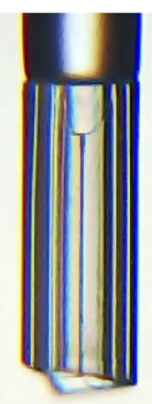

(i)

Fig. 4. Evolution of menisci position inside the sensing head during the process of acetone evaporation (see Media 1).

the effective RI differences due to the environment when light propagates in the SCF core.

Before dipping [phase (a)], all reflections have high values, and light does not interact with any fluid. This translates into a high stable value, as indicated in Fig. 3. Immediately after dipping [phase (b)], most light couples to the external environment, and a strong signal drop $\Delta$ is observed. Considering only the changes in $R_{1}$ between phases (a) and (b), one would expect signal drops of the order of $10 \log _{10}(3.4 / 0.1) \approx 15.3 \mathrm{~dB}$ for acetone and $10 \log _{10}(3.4 / 0.06) \approx 17.5 \mathrm{~dB}$ for IPA. These values are similar to the measured $\Delta$ 's, and the small differences between them may be due to our simplistic calculation that just considers light reflected at the interface and ignores backpropagating light reflected at $R_{2}$ and $R_{3}$ as well as coupling losses between the SMF and the SCF. When acetone from the collapsed region evaporates, between phases (c) and (d), the signal returns to the high state, since reflection values also return to the initial states.

Thus, with a simplified approach, it was possible to interpret the main features of the sensor's time-response. During phases (c) and (d), smaller signal oscillations are observed. These may be due to spectral phase oscillations caused by the interaction between the evanescent field and remaining acetone inside the SCF holes that change the effective RI or due to temperature variations from the evaporation process, since the sensing head was found to have a temperature sensitivity of $(12.16 \pm$ 0.09 ) $\mathrm{pm} /{ }^{\circ} \mathrm{C}$ (adjusted $r^{2}=0.998$ ) in the range from $20^{\circ} \mathrm{C}$ to $100^{\circ} \mathrm{C}$.

It should be noted that, although in the case of acetone, the sensor seems to quickly recover to the initial state immediately after the evaporation of the collapsed

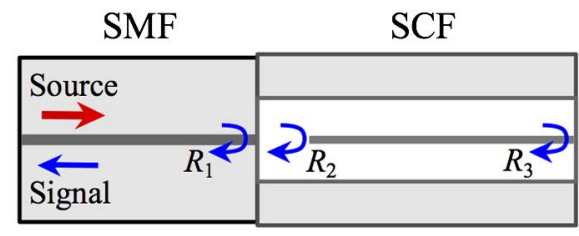

(a)

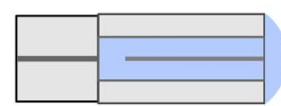

(b)

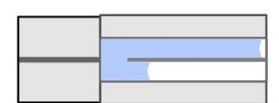

(c)

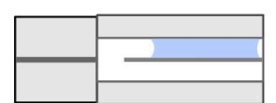

(d)
Fig. 5. (a) Scheme of the sensor structure and (b), (c), and (d) of the main evaporation phases. region, the same does not apply for IPA. In fact, several signal variations are observable for $t>0$, most noticeably the strong dip at the end of evaporation [Fig. 3(e)]. We can attribute these differences to other material properties, such as viscosity, which condition the way menisci move inside the holes and, consequently, how the fluid is positioned throughout evaporation (as seen in Fig. 6). In the case of IPA, it appears that when the first meniscus reaches the collapsed region, the others start propagating from outside in, contrary to what happens in acetone evaporation. As a result, some remaining IPA interferes with the collapsed region before the end of evaporation, provoking the observed signal drop.

In summary, a four-bridge suspended-core microstructured fiber tip, fusion-spliced to a standard SMF, was immersed in acetone and isopropanol, and the evaporation dynamics of each fluid were simultaneously analyzed with an optical microscope and an optical spectrum analyzer. Near the splice, the microstructured fiber is collapsed due to the electric-arc fusion process, which destroys the SCF core and struts, creating microfluidic channels between the fiber holes.

The detailed signal profile evolution and total duration differ between acetone and IPA. These can be considered signatures of each fluid's evaporation properties, directly related to their chemical composition and interactions with the environment. Strong signal variations seem to be related to reflectivity changes in the collapsed region, whereas small signal oscillations are attributed to refractive index changes in the evanescent field of the suspended-core fiber propagating modes.

This configuration could thus be used to detect and study the evaporation of different volatile organic compounds. Contrary to spectroscopy applications, where long sensing heads are preferred to maximize fluid-light interaction, in the case of evaporation monitoring, a short sensor tip should be used to maximize experimental

Table 1. Estimated Reflection Values for Each Evaporation Phase ${ }^{a}$

\begin{tabular}{llccc}
\hline Phase & (a) & (b) & $(\mathrm{c})$ & $(\mathrm{d})$ \\
\hline$R_{1}(\%)$ & 3.4 & $0.1 / 0.06$ & $0.1 / 0.06$ & 3.4 \\
$R_{2}(\%)$ & 3.3 & $0.08 / 0.05$ & $0.08 / 0.05$ & 3.3 \\
$R_{3}(\%)$ & 3.3 & $0.08 / 0.05$ & 3.3 & 3.3 \\
\hline
\end{tabular}

${ }^{a}$ The phases are related to those indicated in Fig. 5 . For (b) and (c), the values were calculated for acetone and IPA, respectively. 


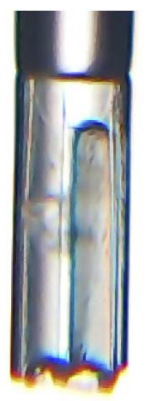

(a)

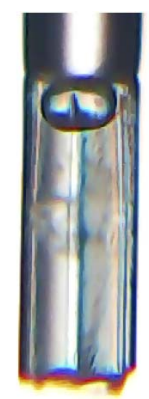

(b)

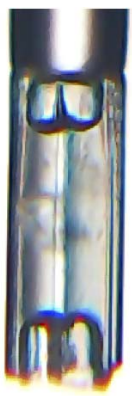

(c)

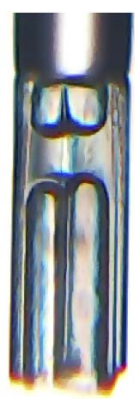

(d)

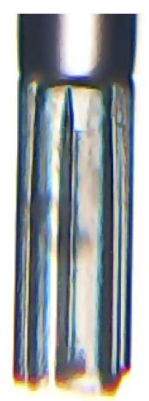

(e)
Fig. 6. Evolution of menisci position inside the sensing head during the process of IPA evaporation (see Media 2).

repeatability, reducing the risk of air bubble formation and facilitating the filling process. Shorter sensors also result in low-volume sensing solutions, which make the proposed configuration useful for industrial or environmental process monitoring. Also, the use of suspended-core fibers, as opposed to direct fabrication of micro-cavities in standard SMFs, makes for sensors with lower cost and easier production and handling.

Finally, in order to further evaluate this configuration's potential as a sensor, future work should include the study of a wider array of volatile compounds. By identifying the main signal components in the evaporation response, this simple sensing setup could be used to distinguish between different fluids and potentially recognize mixtures of volatile compounds.
This work was financed by the FCT, Fundação para a Ciência e Tecnologia (Portuguese Foundation for Science and Technology) and by the ERDF (European Regional Development Fund) through COMPETE Programme (Operational Programme for Competitiveness) within project FCOMP-01-0124-FEDER-037281; ON.2, O Novo Norte (Northern Portugal Regional Operational Programme), under the National Strategic Reference Framework, within project NORTE-07-0124-FEDER000058; and the INTERREG SUDOE Programme (South West Europe Territorial Cooperation Programme) within project "ECOAL-MGT-Ecological Management of Coal Waste Piles, SOE3/P2/P714".

\section{References}

1. T. M. Monro, S. Warren-Smith, E. P. Schartner, A. François, S. Heng, H. Ebendorff-Heidepriem, and S. Afshar, Opt. Fiber Technol. 16, 343 (2010).

2. A. S. Webb, F. Poletti, D. J. Richardson, and J. K. Sahu, Opt. Eng. 46, 010503 (2007).

3. C. M. B. Cordeiro, M. A. R. Franco, G. Chesini, E. C. S. Barretto, R. Lwin, C. H. Brito Cruz, and M. C. J. Large, Opt. Express 14, 13056 (2006).

4. O. Frazão, R. M. Silva, M. S. Ferreira, J. L. Santos, and A. B. Lobo Ribeiro, Photonic Sens. 2, 118 (2012).

5. T. G. Euser, J. S. Y. Chen, M. Scharrer, P. S. J. Russell, N. J. Farrer, and P. J. Sadler, J. Appl. Phys. 103, 103108 (2008).

6. E. Preter, B. Preloznik, V. Artel, C. N. Sukenik, D. Donlagic, and A. Zadok, Sensors 13, 15261 (2013). 